\title{
A comprehensive assessment protocol including patient reported outcomes, physical tests, and biological sampling in newly diagnosed patients with head and neck cancer: is it feasible?
}

\author{
Annette J. van Nieuwenhuizen • Laurien M. Buffart • Jan H. Smit • \\ Ruud H. Brakenhoff • Boudewijn J. M. Braakhuis • Remco de Bree • \\ C. René Leemans • Irma M. Verdonck-de Leeuw
}

Received: 22 April 2014 / Accepted: 21 July 2014 / Published online: 12 August 2014

(C) The Author(s) 2014. This article is published with open access at Springerlink.com

\begin{abstract}
Purpose Large cohort studies are needed taking into account cancer-related, personal, biological, psychobehavioral, and lifestyle-related factors, to guide future research to improve treatment and supportive care. We aimed to evaluate the feasibility of a comprehensive baseline assessment of a cohort study evaluating the course of quality of life (QoL).

Methods Newly diagnosed head and neck cancer (HNC) patients were asked to participate. Assessments consisted of questionnaires (635 items), a home visit (including a psychiatric interview, physical tests, and blood and saliva collection), and tissue collection. Representativeness of the study sample was evaluated by comparing demographics, clinical factors, depression, anxiety, and QoL between responders and non-
\end{abstract}

Meetings at which this manuscript was presented: International PsychoOncology Society, 15th World Congress of Psycho-Oncology, Poster session, 6-8 November 2013, Rotterdam, the Netherlands

A. J. van Nieuwenhuizen • R. H. Brakenhoff • B. J. M. Braakhuis •

R. de Bree $\cdot$ C. R. Leemans $\cdot$ I. M. Verdonck-de Leeuw $(\bowtie)$

Department of Otolaryngology/Head and Neck Surgery, VU

University Medical Center, P.O. Box 7057, 1007 MB Amsterdam,

The Netherlands

e-mail: im.verdonck@vumc.nl

\section{M. Buffart}

Department of Epidemiology and Biostatistics and the EMGO Institute for Health and Care Research, VU University Medical Center, P.O. Box 7057, 1007 MB Amsterdam, The Netherlands

\section{J. H. Smit}

Department of Psychiatry, Neuroscience Campus Amsterdam and EMGO Institute for Health and Care Research, VU University Medical Center, A.J. Ernststraat 1187, 1081 BJ

Amsterdam, The Netherlands responders. Feasibility was evaluated covering the number of questions, time investment, intimacy, and physical burden. Results During the inclusion period (4 months), 15 out of 26 (60\%) patients agreed to participate. Less women participated, $13 \%$ in responders group versus $63 \%$ in non-responders group $(p=0.008)$. No other differences were found between responders and non-responders. Responders completed more than $95 \%$ of the questionnaires' items and rated the number of questions, time investment and intimacy as feasible, and the physical and psychological burden as low. It took on average $3 \mathrm{~h}$ to complete the questionnaires and $1.5 \mathrm{~h}$ for the home visit. Conclusions This study reveals that a comprehensive assessment including various questionnaires, physical measurements, and biological assessments is feasible according to patients with newly diagnosed HNC. A large prospective cohort study has started aiming to include $739 \mathrm{HNC}$ patients and their informal caregivers in the Netherlands.

Keywords Head and neck neoplasm $\cdot$ Health-related quality of life $\cdot$ Physical examination $\cdot$ Pilot study $\cdot$ Feasibility

\section{Introduction}

Worldwide, each year, head and neck cancer (HNC) accounts for more than 633,000 new cases and over 355,000 deaths [1]. In the Netherlands, the incidence of $\mathrm{HNC}$ increased from 2,474 in 2001 to 2,970 in 2011 [http://www. cijfersoverkanker.nl/. 2013. Ref Type: Online Source], mainly due to aging, increased tobacco consumption by females in the 1980s, and an increasing number of human papilloma virus-related oropharyngeal carcinomas [http:// data.euro.who.int/hfadb. 2014. Ref Type: Online Source, 2, 
3]. Current 5-year survival rate of patients with advanced $\mathrm{HNC}$ in the Netherlands is approximately $60 \%$ [http://www. cijfersoverkanker.nl/. 2013. Ref Type: Online Source]. For certain subsites, e.g., oropharyngeal carcinoma, survival is improving [4]. Due to the increasing incidence and improved survival rates, more patients with $\mathrm{HNC}$ have to cope with various physical and psychosocial problems associated with the cancer diagnosis and treatment, such as decreased general and mental health, oral dysfunction, swallowing and speech problems, and emotional distress, severely compromising health-related quality of life (HRQoL) [5-12]. Compared to other types of cancer, including breast, colon, and prostate cancers, HNC patients report high levels of distress [13]. At the same time, an increasing number of studies suggest that HRQoL has prognostic value for survival [14-20]. However, most previous studies on HRQoL and survival in HNC patients had some limitations related to relatively small sample sizes, the focus on specific subsites of HNC, adjustment for only a few confounders (e.g., lifestyle, demographic, and clinical characteristics), or inclusion of only a few aspects of HRQoL (16-25). Furthermore, little is known about the course of HRQoL of patients with $\mathrm{HNC}$ and its determinants across the cancer trajectory. Previous studies showed that several domains of HRQoL, including general health, mental health, physical function, appearance, employment, and social functioning declined during and immediately after treatment and improved after 6 months $[5,6$, 10-12, 21-24]. Studies including long-term follow-up showed that HRQoL stabilized 1 year after treatment and was not significantly different from baseline levels at 5-year follow-up [5-7, 23, 24]. However, Mehanna et al. [12] reported 10 years after diagnosis, significantly lower HRQoL scores than before treatment, which was recently confirmed by Oskam et al. [25].

A recent review among patients with HNC [26] showed that the majority of studies examining the course of HRQoL over time were limited by their retrospective study design, their focus on only a few aspects of HRQoL instead of all domains [27], the use of patient-reported outcomes (PROs) only, the small sample size, and lack of pre-treatment measurements of HRQoL [28]. In addition, studies examining the association between HRQoL and survival lacked to adjust for all relevant confounders and different study designs were used [29]. Therefore, there is need for a large multi-institutional prospective cohort study evaluating the course of HRQoL in patients with $\mathrm{HNC}$ and its relation with survival integrating all relevant cancer-related, personal, genetic, biological, psychobehavioral, physical, lifestyle-related, and social factors. Comprehensive insight in all these factors assessed in a standardized manner is necessary to unravel these complex associations. A study of this magnitude has never been carried out among patients with $\mathrm{HNC}$, and it is unclear whether it is feasible to conduct such an extensive objective assessment of physical and cognitive function, lifestyle, a psychiatric interview, and collection of blood, saliva, and tumor tissue, in addition to a large number of PROs. Thus, the aim of the present study is to assess the feasibility of a comprehensive baseline assessment among patients with HNC.

\section{Materials and methods}

Sample and setting

As part of clinical routine in our institution, all newly diagnosed HNC patients are asked to fill out questionnaires on HRQoL and emotional distress during their first visit via OncoQuest [30]. OncoQuest is a touch screen computer system to monitor HRQoL in clinical practice and includes three questionnaires: the European Organization for Research and Treatment of Cancer (EORTC), Quality of Life Questionnaire core module (QLQ-C30), [31, 32], EORTC Head and Neck Module (HN35) [33], and Hospital Anxiety and Depression Scale (HADS) [34].

The 30-item EORTC QLQ-C30 includes a global quality of life scale, five function scales regarding physical, role, emotional, cognitive, and social functioning, three symptom scales (nausea and vomiting, fatigue, and pain), and six single items related to dyspnea, insomnia, loss of appetite, constipation, diarrhea, and financial difficulties [31, 35]. The 35-item EORTC QLQ-HN35 is a tumor-specific module addressing symptoms specifically associated with $\mathrm{HNC}$, including pain, swallowing, senses, speech, social eating, social contact, and sexuality, as well as ten single items covering problems with teeth, dry mouth, sticky saliva, cough, opening the mouth wide, weight loss, weight gain, use of nutritional supplements, feeding tubes, and painkillers [32]. The HADS is a 14-item scale for measuring emotional distress and includes a total scale and an anxiety (HADS-A) and depression (HADS-D) subscale [34]. A total score of $>15$ is used as an indicator of a high psychological distress. For the subscales, cutoff points of $\geq 8$ are indicators of high levels of anxiety or depression.

From January to mid-April 2012, every new patient with HNC was screened for eligibility for the current feasibility study. Patients presenting with oral, oropharyngeal, hypopharyngeal, and laryngeal cancers and patients with neck metastasis of unknown primary tumor with proven squamous cell histology (aged 18 years or older, treated with curative intent), who were able to write, read, and speak Dutch were eligible. Patients suffering from severe psychiatric comorbidities (e.g., schizophrenia, Korsakov's syndrome, and severe dementia) were excluded. Eligible patients were asked to participate in this feasibility study by the treating surgeon, and subsequently, the research physician provided more detailed information about the study and handed out written information. Non-participants were asked for their reason for not participating. All patients signed an informed consent 
statement prior to participation. The Institutional Review Board of the VU University Medical Center approved the study.

\section{Procedures and assessments}

After all eligible patients filled out the three questionnaires (EORTC QLQ C30, EORTC HN35, HADS) as clinical procedure, study participants filled out the comprehensive baseline assessment, consisting of 36 questionnaires and in total 762 items, which took place before the start of treatment. The questionnaires consisted variable subdomains, including general and disease-specific QoL, cancer-related, personal, psychobehavioral, physical, lifestyle-related, social factors, and health care costs (Table 1). According to patient's preference, the PROs were sent by postal mail, or a link was emailed to fill out the PROs via internet. Subsequently, the research physician visited the patients at their homes or in the hospital according to preference of the patients, to conduct a psychiatric interview, physical tests, and collection of blood. At the end of the visit, patients were instructed to collect five saliva samples during the same evening and next morning and to wear an accelerometer (Actitrainer, Actigraph, Fort Walton Beach, Florida) for the next 7 days. Three to 5 days after the home visit, a telephone interview was conducted to assess diet using a 24-h recall. Tumor tissue was gathered during the participants' panendoscopic procedure, which is performed as a part of the diagnostic workup. This procedure aims to determine the field of surgery and to investigate the presence of other tumors. During this panendoscopy, a supplemental biopsy was taken for the current study besides the diagnostic tissue collection for the pathology department. An overview of all outcome measures included in the baseline assessment protocol is presented in Table 1. The assessment protocol was developed in collaboration with the coordinator of the Netherlands Study of Depression and Anxiety (NESDA) study [33]. NESDA is a large prospective cohort study which aims to describe the long-term course and consequences of depressive and anxiety disorders.

\section{Outcome measures}

The primary outcome of this study was the feasibility of the baseline assessment protocol, as evaluated by representativeness of the study population, achievability of baseline assessments, and accuracy of the protocol.

Representativeness was assessed by the following questions:

- What percentage of eligible patients is willing to participate?

- What are the main reasons for not participating?
- Are there differences between participants and nonparticipants regarding age, gender, diagnosis, comorbidity, stage, treatment, HRQoL, and emotional distress?

Achievability was evaluated by the following items:

- A study-specific questionnaire consisting of four-point Likert scales (not feasible, a bit feasible, quit feasible, very much feasible) covering the number of items, time investment, intimacy and burden of the PROs, and the home visit.

- The number of items successfully completed was registered as well as the time needed to complete the PROs, as estimated by patients and the home visits as measured by a research physician.

Accuracy of the assessment protocol was evaluated using the following questions:

- Is the protocol clear to other assessors (i.e., two experienced fieldworkers and a coordinator from the NESDA study)?

- Is the assessment protocol complete, clear, and accurate according to the research physician (A.N.)?

Statistical analysis

Statistical analyses were performed using Statistical Package for the Social Sciences (SPSS) 20.0 (SPSS Inc., Chicago, IL). Descriptive statistics (mean/standard deviation (SD)/proportions) were generated for demographic and clinical characteristics, emotional distress, and HRQoL and questions on representativeness and achievability.

Differences in age, gender, diagnosis, comorbidity, stage, treatment, emotional distress, and HRQoL between participants and non-participants were tested with Mann-Whitney $U$ test or $\chi^{2}$ test. For the comparison of emotional distress and HRQoL, we used results from the OncoQuest database.

\section{Results}

Representativeness

During a time period of 14 weeks, 26 eligible patients were asked, of whom 15 (58\%) were willing to participate (Fig. 1). The main reason for not participating was the high burden of recently being diagnosed with cancer, leaving no room for additional inconvenience $(n=8 ; 30 \%)$. However, all of these patients indicated that they would have been interested in participation at another point in time. Other reasons for non- 
Table 1 Overview of all outcome measures included in the assessment protocol: patient-reported outcome, (home) visit (physical tests and interviews), and biological and clinical factors

\begin{tabular}{lll}
\hline Outcomes & Measurement instrument & Number of \\
& & items
\end{tabular}

Patient-reported outcome

Quality of life

Generic

Disease-specific

EuroQuol-5D (EQ-5D)

Tumor-specific

European Organisation for Research and Treatment of Cancer Quality of Life

Questionnaire-core 30 (EORTC QLQ-C30)

European Organisation for Research and Treatment of Cancer Quality of Life 35

Speech

Swallowing

Questionnaire module, Head and Neck 35 (EORTC QLQ-HN35)

Speech Handicap Index (SHI)

Swallowing QoL Questionnaire (SwalQoL) $\quad 47$

Shoulder

Shoulder Disability Questionnaire (SDQ)

47
16

Hearing

Malnutrition

Caron questionnaire on hearing

19

Short Nutritional Assessment Questionnaire (SNAQ)

Cancer-related factors

Comorbidity

Adult comorbidity evaluation (ACE-27)

Personal factors

Personality

Neuroticism-Extroversion-Openness-Five factor inventory (NEO-FFI)

Locus of control

Pearlin and Schooler mastery scale (PSMS)

Coping

Self-efficacy

Well-being

Physical appearance

Mental Adjustment to Cancer scale (MAC)

Generalized self-efficacy scale (GES)

Posttraumatic Growth Inventory (PTGI)

Body Image Scale (BIS)

Life events

Brugha Questionnaire

Coping

Utrechtse Coping List (UCL)

Psychobehavioral factors

Severity anxiety/depression

Hospital Anxiety and Depression Scale (HADS)

Fatigue

Multidimensional Fatigue Inventory (MFI)

Fatigue

Sleep quality

Fatigue Quality List (FQL)

Pittsburgh Sleep Quality Index (PSQI) $\quad 15$

Fear of recurrence

Cancer Worry Scale (CWS)

Cognition

Cognitive Failure Questionnaire (CFQ)

Physical factors

Sexual function

Males

Females

Females

Lifestyle-related factors

Alcohol intake

Alcohol dependence

Drug use

Smoking

Nicotine dependence

Physical activity

Leisure

Social factors

Social support

Participation

International index of Erectile Function (IIEF)

19

Female Sexual Function Index (FSFI)

Study-specific questionnaire on fertility

Study-specific questions

13

Study-specific questions

Study-specific questions

Study-specific questions

Study-specific questions

Physical Activity Scale for the Elderly (PASE) 31

Study-specific questions

Social Support List Interactions (SSL-I12)

Health care use/costs

Need and use care 
Table 1 (continued)

\begin{tabular}{|c|c|c|}
\hline Outcomes & Measurement instrument & $\begin{array}{l}\text { Number of } \\
\text { items }\end{array}$ \\
\hline Costs & $\begin{array}{l}\text { Trimbos and iMTA questionnaire on Costs associated with Psychiatric } \\
\text { illness (TiC-P): Adapted version for HNC }\end{array}$ & 30 \\
\hline \multicolumn{3}{|l|}{ (Home) visit } \\
\hline \multicolumn{3}{|l|}{ Personal factors } \\
\hline Demographic & Standard questions & 23 \\
\hline Socioeconomic status/literacy & Standard questions & 27 \\
\hline \multicolumn{3}{|l|}{ Psychobehavioral factors } \\
\hline Presence MDD & Composite international diagnostic interview (CIDI)—Major Depressive Disorder (MDD) & Variable \\
\hline Anxiety disorder & Composite international diagnostic interview (CIDI)-Anxiety disorders (GAD, SOC, PAN, AG) & Variable \\
\hline Pain & Brief Pain Inventory (BPI) & 13 \\
\hline \multicolumn{3}{|l|}{ Physical factors } \\
\hline Speech quality & Speech recording (perceptual and objective analyses) & n.a. \\
\hline Pulmonary function & Peak flow & n.a. \\
\hline Strength-upper extremity & JAMAR handgrip dynamometer & n.a. \\
\hline Strength-lower extremity & 30s chair stand test & n.a. \\
\hline Cardiorespiratory fitness & Chester Step test & n.a. \\
\hline Body composition & Height, weight, body mass index, waist+hip circumference, thickness of four skin folds & n.a. \\
\hline Blood pressure & Systolic and diastolic blood pressure & n.a. \\
\hline Visual motor processing speed & Trail making test part $\mathrm{A}$ & n.a. \\
\hline Executive functioning & Trail making test part B & n.a. \\
\hline Activity monitoring & Accelerometer & n.a. \\
\hline Food & 24-h recall & n.a. \\
\hline \multicolumn{3}{|l|}{ Social factors } \\
\hline Loneliness & De Jong-Gierveld Loneliness Scale & 11 \\
\hline \multicolumn{3}{|l|}{ Health care use/costs } \\
\hline Work productivity & Productivity and disease questionnaire (PRODISQ) & 14 \\
\hline \multicolumn{3}{|l|}{ Biological and clinical factors } \\
\hline \multicolumn{3}{|l|}{ Cancer-related factors } \\
\hline Cancer location/stage & Standard questions, from medical record & n.a. \\
\hline Treatment modality & Standard questions, from medical record & n.a. \\
\hline \multicolumn{3}{|l|}{ Biological factors } \\
\hline Tumor markers & Tumor tissue & n.a. \\
\hline Biomarkers & Blood & n.a. \\
\hline General laboratory & Blood & n.a. \\
\hline DNA & Blood & n.a. \\
\hline Proteomics & Blood & n.a. \\
\hline Gene expression (RNA) & Blood & n.a. \\
\hline
\end{tabular}

$M D D$ major depressive disorder, n.a. not applicable, $A D$ generalized anxiety disorder, $S O C$ social phobia, $P A N$ panic disorder, $A G$ agoraphobia

participation were not willing to participate in any research project $(n=2 ; 8 \%)$ or not willing to participate in this specific study protocol $(n=1 ; 4 \%)$.

Except for gender, no differences in demographic and clinical characteristics, emotional distress, and HRQoL were found between participants and non-participants. The proportion of women among non-participants $(64 \%)$ was higher than among participants $(13 \%), p=0.008$ (Table 2 ).
One participant (7\%) and two non-participants (18\%) did not fill out OncoQuest, and consequently, their data on HRQoL and psychological distress were missing.

Achievability

All participating patients filled out the questionnaires with 95 $99 \%(n=8)$ to $100 \%(n=7)$ of the items completed. Two 
Fig. 1 Flowchart of all eligible patients and the reasons for non-participation

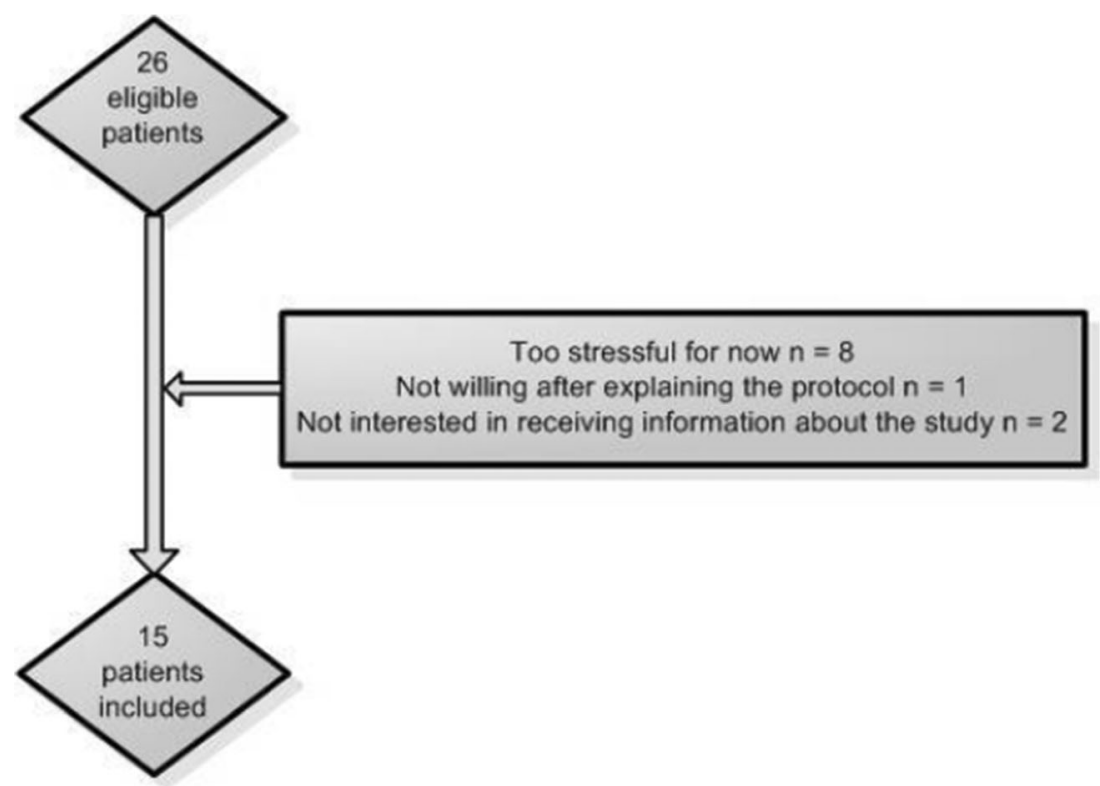

patients did not fill out the questionnaire on sexuality because they were not sexually active. No problems were detected with other questionnaires. The majority of the patients $(80 \%)$ preferred the pen-and-paper version over the internet-based method. Completing the PROs took on average 167 min (range 100-270). All patients filled out the PROs within 1 week. One patient needed $270 \mathrm{~min}$ to complete the PROs due to concentration problems. Compared to the other patients, this was exceptionally long (range without this particular patient 100-210 $\mathrm{min}$ ).

Most patients $(n=12)$ preferred the research physician to conduct the interview, physical tests, and blood collection during a home visit. Assessments of the other three patients were conducted during a hospital visit. The visits took on average 100 min (range 60-145).

Some of the physical tests and biological sample collections could not be performed (Table 3). The Chester step test was not conducted in three patients due to physical impairments such as an amputated leg, cardiac history, or severe mobility problems. Accelerometer data of six patients were missing due to the following: surgery within a few days after the baseline measurement $(n=1)$, a percutaneous endoscopic gastrostomy (PEG) tube placement within a few days after baseline assessment $(n=2)$, wheelchair dependence $(n=1)$, crutches dependence in daily life $(n=1)$, and losing the accelerometer $(n=1)$. All other physical tests were completed by all patients. The cognitive test and the psychiatric interview were conducted in all patients without experiencing any problems. Dietary telephonic interview at the end of the assessments were taken in $11(73 \%)$ patients. Four interviews were missed because treatment already started. Blood and saliva samples were collected in 13 patients $(87 \%)$ patients; two patients refused to give blood samples, and two patients did not return their saliva samples. Tumor tissue from eight participants
(53\%) was collected during panendoscopy at the VU University Medical Center. Reasons for not collecting supplemental biopsies were as follows: unknown primary tumor $(n=2)$, no extra biopsies during panendoscopy because of immediate surgery $(n=3)$, biopsy already taken in outpatient clinic $(n=$ $1)$, and unknown $(n=1)$.

Patients evaluated the number of items, the time investment and personal or intimate character of the PROs, and the physical tests as feasible to very much feasible (Table 4). Regarding intimacy of questions, one patient found a questionnaire on sexuality too intimate and therefore rated the item intimacy as "a bit feasible" for intimacy. One patient found the time investment of the home visit too long. Due to the presence of a child, this home visit took much longer compared to the other patients (145 min).

\section{Protocols}

Generally, the research physician reported the home visits to be very pleasant. Concentration problems were present in two patients according to the research physician. Another patient was somewhat long-winded and expanding to various topics during the (psychiatric) interview and had to be redirected to the questions continuously. No adverse events occurred during the visits. Furthermore, the protocols were independently judged as clear by the research physician, two other experienced fieldworkers, and a research coordinator.

\section{Discussion}

This pilot study showed that it is feasible to conduct a comprehensive baseline assessment compromising a large number of PROs, interviews, physical tests, and biological sample 
Table 2 Differences in demographic and clinical characteristics, psychological distress, and health-related quality of life between participants and nonparticipants

\begin{tabular}{|c|c|c|c|}
\hline Characteristics & Participants $(n=15)$ & Non-participants $(n=11)$ & Difference ( $p$ value) \\
\hline Gender, $n(\%)$ male & $13(87)$ & $4(36)$ & 0.008 \\
\hline Age, mean $\pm \mathrm{SD}$ (range) (years) & $63 \pm 12(40-80)$ & $62 \pm 8(52-78)$ & 0.926 \\
\hline Tumor location, $n(\%)$ & & & 0.986 \\
\hline Oral cavity & $4(27)$ & $3(27)$ & - \\
\hline Oropharynx & $5(33)$ & $3(27)$ & - \\
\hline Hypopharynx & $0(0)$ & $0(0)$ & - \\
\hline Larynx & $5(33)$ & $4(36)$ & - \\
\hline Unknown primary & $1(7)$ & $1(9)$ & - \\
\hline Disease stage, $n(\%)$ & & & 0.749 \\
\hline $\mathrm{I}$ & $2(13)$ & $2(18)$ & - \\
\hline II & $1(7)$ & $1(9)$ & - \\
\hline III & $5(33)$ & $3(27)$ & - \\
\hline IV & $7(46)$ & $5(45)$ & - \\
\hline Type of treatment, $n(\%)$ & & & 0.486 \\
\hline CHRT & $8(53)$ & $4(36)$ & - \\
\hline RT & $4(27)$ & $2(18)$ & - \\
\hline SURG & $1(7)$ & $3(27)$ & - \\
\hline $\mathrm{TOE}+\mathrm{SN}$ & $2(13)$ & $2(18)$ & - \\
\hline \multicolumn{4}{|l|}{ Comorbidity, $n(\%)$} \\
\hline None & $4(27)$ & $3(27)$ & 0.683 \\
\hline Mild & $3(20)$ & $3(27)$ & - \\
\hline Moderate & $7(47)$ & $3(27)$ & - \\
\hline \multirow[t]{2}{*}{ Severe } & $1(7)$ & $2(18)$ & - \\
\hline & Participant $(n=14)$ & Non-participant $(n=9)$ & \\
\hline \multicolumn{4}{|l|}{ HADS } \\
\hline Total score, mean \pm SD & $10 \pm 8$ & $11 \pm 5$ & 0.587 \\
\hline Depression score $\geq 8, n(\%)$ & $3(21 \%)$ & $1(11 \%)$ & 0.546 \\
\hline Anxiety score $\geq 8, n(\%)$ & $3(21 \%)$ & $4(44 \%)$ & 0.262 \\
\hline Total score $>15, n(\%)$ & $3(21 \%)$ & $2(22 \%)$ & 0.966 \\
\hline \multicolumn{4}{|l|}{ EORTC QLQ-C30, mean \pm SD } \\
\hline Global quality of life & $78 \pm 15$ & $64 \pm 29$ & 0.145 \\
\hline Physical function & $87 \pm 15$ & $81 \pm 23$ & 0.480 \\
\hline Role function & $88 \pm 24$ & $74 \pm 30$ & 0.229 \\
\hline Emotional function & $60 \pm 22$ & $69 \pm 20$ & 0.296 \\
\hline Cognitive function & $88 \pm 21$ & $94 \pm 8$ & 0.402 \\
\hline Social function & $92 \pm 18$ & $85 \pm 28$ & 0.508 \\
\hline
\end{tabular}

$S D$ standard deviation, CHRT chemoradiation therapy, EORTC QLQ C30 European Organization for Research and Treatment of Cancer Quality of Life Questionnaire Core 30, HADS Hospital Anxiety and Depression Scale, $R T$ radiotherapy, $S U R G$ surgery, $T O E+S N$ transoral excision and sentinel node procedure

collection among newly diagnosed $\mathrm{HNC}$ patients. We found the study sample to be a representative reflection of patients with $\mathrm{HNC}$, and the achievability of the assessment protocol was high.

\section{Representativeness}

The present study showed that $58 \%$ of newly diagnosed HNC patients were willing to participate in a comprehensive assessment. Our response rate was somewhat lower compared to $76-97 \%$ reported in the other prospective cohort studies on HRQoL in HNC patients [5, 7, 10, 36, 37]. Differences in response rate may be related to the large number of questionnaires included, since only one to three questionnaires were included in the previously mentioned studies. Taking into account our comprehensive assessments, we consider our response rate to be acceptable. Of all eligible patients, $30 \%$ 
Table 3 Achievability of physical tests and biological sample collection

\begin{tabular}{|c|c|c|}
\hline $\begin{array}{l}\text { Physical } \\
\text { assessments }\end{array}$ & $\begin{array}{l}\text { Percentage } \\
\text { performed }\end{array}$ & $\begin{array}{l}\text { Reasons for not performing } \\
\text { physical assessments }\end{array}$ \\
\hline Grip strength & 100 & - \\
\hline 30-s chair stand test & 93 & Amputated leg $(n=1)$ \\
\hline Step test & 80 & $\begin{array}{l}\text { Amputated leg }(n=1) \\
\text { Cardiac history }(n=1) \\
\text { Severe immobility }(n=1)\end{array}$ \\
\hline Accelerometer & 53 & $\begin{array}{l}\text { Amputated leg }(n=1) \\
\text { Severe immobility }(n=1) \\
\text { PEG tube placement }(n=2) \\
\text { Lost accelerometer }(n=2) \\
\text { Surgery, within a few days } \\
\quad \text { after visit }(n=1)\end{array}$ \\
\hline $\begin{array}{l}\text { Biological sample } \\
\text { collection }\end{array}$ & $\begin{array}{l}\text { Percentage } \\
\text { performed }\end{array}$ & $\begin{array}{l}\text { Reasons for not performing } \\
\text { biological assessment }\end{array}$ \\
\hline Blood collection & 87 & Unwillingness $(n=2)$ \\
\hline Saliva collection & 87 & Not returned $(n=2)$ \\
\hline Tissue collection & 53 & $\begin{array}{l}\text { Biopsy already taken }(n=1) \\
\text { No extra biopsies, direct } \\
\text { surgery }(n=3) \\
\text { No extra biopsies, unknown } \\
\text { primary tumor }(n=2) \\
\text { Reason not registered }(n=1)\end{array}$ \\
\hline
\end{tabular}

$P E G$ percutaneous endoscopic gastrostomy

would like to participate in the study but not at this specific moment. The most important argument for not participating was that the protocol seems too burdensome. For a future study, we can now better inform eligible patients on the time investments and feasibility of the protocol. The remaining $12 \%$ of eligible patients were not willing to participate in any study, and it is likely that this proportion will also be missed in a forthcoming cohort study.

In our study, females were found to be less likely to participate, which is in contrast to the pilot study of Hammerlid et al. [38] who found all non-participants to be men. Another prospective study on long-term HRQoL in patients with $\mathrm{HNC}$ found no significant differences between participants and non-participants in demographic and clinical characteristics [7]. Therefore, our under-representativeness of women may be coincidental. On the other hand, it may be related to the higher, but non-significant anxiety levels we found among non-participants, since, in patients with HNC, women are more likely to report higher levels of anxiety than men [39]. However, due to the small sample size, the nonsignificant differences in anxiety between participants and non-participants should be interpreted with caution. Studies among newly diagnosed cancer patients are at risk for selection bias underestimating problems related to emotional distress and HRQoL. Therefore, we will closely monitor differences in main characteristics between responders and nonresponders in a future cohort study.

Achievability

Despite the considerable time investment (average of $270 \mathrm{~min}$ in total), our results showed that almost all patients found the study to be feasible to very much feasible. However, some patients experienced some problems with the questionnaires on sexuality, particularly those who were not sexually active. To avoid unnecessary confrontation with intimate questions in a future cohort study, we will therefore add a remark at the start of this questionnaire that patients can skip this questionnaire if not applicable. Regarding the (home) visits, we noticed that a quiet environment is important to prevent unnecessary delay in conducting the interviews and physical tests.

The home visits were almost fully completed. Blood and saliva was collected in $87 \%$ of the patients which we considered to be acceptable. A relatively large proportion ( $40 \%$ ) of patients did not wear the accelerometer due to various reasons, of which some were largely unavoidable such as amputated leg and crutches dependence, whereas others may be prevented or (rapidly) solved. In this study, we did not give an accelerometer to patients undergoing a PEG tube placement which potentially hampers physical activities for a certain period of time. However, it seems that the patients may be

Table 4 Feasibility of the questionnaires and home visit

\begin{tabular}{|c|c|c|c|c|}
\hline & Not feasible & A bit feasible & Feasible & Very much feasible \\
\hline \multicolumn{5}{|l|}{ Questionnaires, $n(\%)$} \\
\hline Number of items & - & - & $12(86)$ & $2(14)$ \\
\hline Time investment & - & - & $6(40)$ & $8(57)$ \\
\hline Personal or intimate character of questions & - & $1(7)$ & $7(50)$ & $6(43)$ \\
\hline \multicolumn{5}{|l|}{ Home visits, $n(\%)$} \\
\hline Number of questions & - & - & $3(21)$ & $11(79)$ \\
\hline Time investment & $1(7)$ & - & $4(29)$ & $9(64)$ \\
\hline Personal or intimate character of questions & - & - & $2(14)$ & $12(86)$ \\
\hline Burden of physical assessments & - & - & $2(14)$ & $12(86)$ \\
\hline
\end{tabular}


able to wear an accelerometer. Furthermore, a new device may quickly be sent to patients who lost their device. Tissue collection was successful in $53 \%$ of the participants. In most cases, supplemental tumor biopsies for this study were not taken due to logistical reasons including immediate surgical treatment of tumor, biopsies already taken in outpatient clinic, or because of an unknown primary tumor. In this pilot study, we did not collect tumor tissue samples from the surgical specimen from the pathology department, but we are planning to do so in a future study if tumor tissue is available.

\section{Strengths and limitations}

To our knowledge, this pilot study is the first to evaluate a comprehensive assessment protocol of this extent in newly diagnosed HNC patients before treatment. In addition, the inclusion of objective physical tests and biological sample collection in addition to PROs is a strength of this study. Another strength of this study was the ability to compare data on HRQoL and psychological distress between participants and non-participants, providing a thorough investigation of the representativeness of the included study sample. This study was limited by its focus on baseline assessment only, and we did not collect data on the feasibility of follow-up measurements. However, other longitudinal studies on HRQoL in patients with $\mathrm{HNC}$ reported acceptable dropout rates varying between 6 and $14 \%[7,36]$. Whether dropout rates will be similar in the forthcoming cohort study remains unclear. Furthermore, while almost all single questionnaires were validated, it is unclear whether assessment of multiple single valid questionnaires impacts the validity. A previous study examining the influence of the structure of questionnaires on response outcomes showed that changes in order of questionnaires did not substantially affect the outcomes [40]. In the present study, assessments were conducted in a hierarchical order, starting with the main outcome measure health-related quality of life, following by questionnaires assessing covariates.

In conclusion, this study showed that it is feasible to conduct a comprehensive assessment protocol including PROs, interviews, physical tests, and collection of biological samples in newly diagnosed HNC patients before the start of treatment. Therefore, we will set up a large multicenter cohort study in patients with HNC evaluating the course of HRQoL over time starting at diagnosis and the relationship between HRQoL and survival, taking into account cancer-related, personal, genetic, biological, psychobehavioral, physical, lifestyle-related, and social factors, the Netherlands Quality of life and Biomedical Cohort studies in cancer, Head and Neck (NET-QUBIC_HNC).

Acknowledgments We would like to thank the participating patients for their contribution to this study.
Conflict of interest There was no financial conflict of interest. We have full control of all primary data, and we agree to allow the journal to review the data if requested.

Funding No funding for this work was received.

Open Access This article is distributed under the terms of the Creative Commons Attribution Noncommercial License which permits any noncommercial use, distribution, and reproduction in any medium, provided the original author(s) and the source are credited.

\section{References}

1. Ferlay J, Shin HR, Bray F, Forman D, Mathers C, Parkin DM (2010) Estimates of worldwide burden of cancer in 2008: GLOBOCAN 2008. Int J Cancer 127(12):2893-2917

2. Braakhuis BJ, Visser O, Leemans CR (2009) Oral and oropharyngeal cancer in The Netherlands between 1989 and 2006: Increasing incidence, but not in young adults. Oral Oncol 45(9):e85-e89

3. Rietbergen MM, Leemans CR, Bloemena E, Heideman DA, Braakhuis BJ, Hesselink AT, et al (2012) Increasing prevalence rates of HPV attributable oropharyngeal squamous cell carcinomas in the Netherlands as assessed by a validated test algorithm. Int $\mathrm{J}$ Cancer

4. Braakhuis BJ, Leemans CR, Visser O (2014) Incidence and survival trends of head and neck squamous cell carcinoma in the Netherlands between 1989 and 2011. Oral Oncol 50(7):670-5

5. Abendstein H, Nordgren M, Boysen M, Jannert M, Silander E, Ahlner-Elmqvist $\mathrm{M}$ et al (2005) Quality of life and head and neck cancer: a 5 year prospective study. Laryngoscope 115(12):21832192

6. Bjordal K, Ahlner-Elmqvist M, Hammerlid E, Boysen M, Evensen JF, Biorklund A et al (2001) A prospective study of quality of life in head and neck cancer patients. Part II: Longitudinal data. Laryngoscope 111(8):1440-1452

7. de Graeff A, de Leeuw JR, Ros WJ, Hordijk GJ, Blijham GH, Winnubst JA (2000) Long-term quality of life of patients with head and neck cancer. Laryngoscope 110(1):98-106

8. Funk GF, Karnell LH, Christensen AJ (2012) Long-term healthrelated quality of life in survivors of head and neck cancer. Arch Otolaryngol Head Neck Surg 138(2):123-133

9. Goldstein DP, Hynds KL, Christensen AJ, Funk GF (2007) Healthrelated quality of life profiles based on survivorship status for head and neck cancer patients. Head Neck 29(3):221-229

10. Hammerlid E, Bjordal K, Ahlner-Elmqvist M, Boysen M, Evensen JF, Biorklund A et al (2001) A prospective study of quality of life in head and neck cancer patients. Part I: at diagnosis. Laryngoscope 111(4 Pt 1):669-680

11. Hammerlid E, Silander E, Hornestam L, Sullivan M (2001) Healthrelated quality of life three years after diagnosis of head and neck cancer - a longitudinal study. Head Neck 23(2):113-125

12. Mehanna HM, Morton RP (2006) Deterioration in quality-of-life of late (10-year) survivors of head and neck cancer. Clin Otolaryngol 31(3):204-211

13. Carlson LE, Angen M, Cullum J, Goodey E, Koopmans J, Lamont L et al (2004) High levels of untreated distress and fatigue in cancer patients. Br J Cancer 90(12):2297-2304

14. Fang FM, Liu YT, Tang Y, Wang CJ, Ko SF (2004) Quality of life as a survival predictor for patients with advanced head and neck carcinoma treated with radiotherapy. Cancer 100(2):425-432

15. Karvonen-Gutierrez CA, Ronis DL, Fowler KE, Terrell JE, Gruber SB, Duffy SA (2008) Quality of life scores predict survival among patients with head and neck cancer. J Clin Oncol 26(16):2754-2760 
16. Mehanna HM, Morton RP (2006) Does quality of life predict longterm survival in patients with head and neck cancer? Arch Otolaryngol Head Neck Surg 132(1):27-31

17. Meyer F, Fortin A, Gelinas M, Nabid A, Brochet F, Tetu B et al (2009) Health-related quality of life as a survival predictor for patients with localized head and neck cancer treated with radiation therapy. J Clin Oncol 27(18):2970-2976

18. Oskam IM, Verdonck-de LI, Aaronson NK, Kuik DJ, de Bree R, Doornaert P et al (2010) Quality of life as predictor of survival: a prospective study on patients treated with combined surgery and radiotherapy for advanced oral and oropharyngeal cancer. Radiother Oncol 97(2):258-262

19. Osthus AA, Aarstad AK, Olofsson J, Aarstad HJ (2011) Head and neck specific Health Related Quality of Life scores predict subsequent survival in successfully treated head and neck cancer patients: a prospective cohort study. Oral Oncol 47(10):974-979

20. Urba S, Gatz J, Shen W, Hossain A, Winfree K, Koustenis A et al (2012) Quality of life scores as prognostic factors of overall survival in advanced head and neck cancer: analysis of a phase III randomized trial of pemetrexed plus cisplatin versus cisplatin monotherapy. Oral Oncol 48(8):723-729

21. Borghgraef K, Delaere P, Van den BW, Feenstra L (1997) Quality of life in head and neck cancer patients. Acta Otorhinolaryngol Belg 51(2):69-72

22. Chen AM, Jennelle RL, Grady V, Tovar A, Bowen K, Simonin P et al (2009) Prospective study of psychosocial distress among patients undergoing radiotherapy for head and neck cancer. Int J Radiat Oncol Biol Phys 73(1):187-193

23. de Graeff A, de Leeuw J, Ros WJ, Hordijk GJ, Blijham GH, Winnubst JA (2000) Long-term quality of life of patients with head and neck cancer. Laryngoscope 110(1):98-106

24. Hammerlid E, Ahlner-Elmqvist M, Bjordal K, Biorklund A, Evensen J, Boysen M et al (1999) A prospective multicentre study in Sweden and Norway of mental distress and psychiatric morbidity in head and neck cancer patients. Br J Cancer 80(5-6):766-774

25. Oskam IM, Verdonck-de Leeuw IM, Aaronson NK, Witte BI, de Bree R, Doornaert P et al (2013) Prospective evaluation of health-related quality of life in long-term oral and oropharyngeal cancer survivors and the perceived need for supportive care. Oral Oncol 49(5):443-448

26. Verdonck-de Leeuw IM, van Nieuwenhuizen AJ, Leemans CR (2012) The value of quality-of-life questionnaires in head and neck cancer. Curr Opin Otolaryngol Head Neck Surg 20(2):142-147

27. Sayed SI, Elmiyeh B, Rhys-Evans P, Syrigos KN, Nutting CM, Harrington KJ et al (2009) Quality of life and outcomes research in head and neck cancer: a review of the state of the discipline and likely future directions. Cancer Treat Rev 35(5):397-402

28. Quinten C, Coens C, Mauer M, Comte S, Sprangers MA, Cleeland C et al (2009) Baseline quality of life as a prognostic indicator of survival: a meta-analysis of individual patient data from EORTC clinical trials. Lancet Oncol 10(9):865-871

29. Verdonck-de LI, van Nieuwenhuizen A, Leemans CR (2012) The value of quality-of-life questionnaires in head and neck cancer. Curr Opin Otolaryngol Head Neck Surg 20(2):142-147

30. Verdonck-de Leeuw IM, de Bree R, Keizer AL, Houffelaar T, Cuijpers P, van der Linden MH et al (2009) Computerized prospective screening for high levels of emotional distress in head and neck cancer patients and referral rate to psychosocial care. Oral Oncol 45(10):e129-e133

31. Fayers P, Bottomley A (2002) Quality of life research within the EORTC-the EORTC QLQ-C30. European Organisation for Research and Treatment of Cancer. Eur J Cancer 38(Suppl 4):S125-S133

32. Bjordal K, Ahlner-Elmqvist M, Tollesson E, Jensen AB, Razavi D, Maher EJ et al (1994) Development of a European Organization for Research and Treatment of Cancer (EORTC) questionnaire module to be used in quality of life assessments in head and neck cancer patients. EORTC Quality of Life Study Group. Acta Oncol 33(8): 879-885

33. Penninx BW, Beekman AT, Smit JH, Zitman FG, Nolen WA, Spinhoven P et al (2008) The Netherlands Study of Depression and Anxiety (NESDA): rationale, objectives and methods. Int J Methods Psychiatr Res 17(3):121-140

34. Spinhoven P, Ormel J, Sloekers PP, Kempen GI, Speckens AE, Van Hemert AM (1997) A validation study of the Hospital Anxiety and Depression Scale (HADS) in different groups of Dutch subjects. Psychol Med 27(2):363-370

35. Aaronson NK, Ahmedzai S, Bergman B, Bullinger M, Cull A, Duez NJ et al (1993) The European Organization for Research and Treatment of Cancer QLQ-C30: a quality-of-life instrument for use in international clinical trials in oncology. J Natl Cancer Inst 85(5):365-376

36. de Graeff A, de Leeuw JR, Ros WJ, Hordijk GJ, Blijham GH, Winnubst JA (2000) Pretreatment factors predicting quality of life after treatment for head and neck cancer. Head Neck 22(4):398-407

37. Osthus AA, Aarstad AK, Olofsson J, Aarstad HJ (2011) Healthrelated quality of life scores in long-term head and neck cancer survivors predict subsequent survival: a prospective cohort study. Clin Otolaryngol 36(4):361-368

38. Hammerlid E, Bjordal K, Ahlner-Elmqvist M, Jannert M, Kaasa S, Sullivan M et al (1997) Prospective, longitudinal quality-of-life study of patients with head and neck cancer: a feasibility study including the EORTC QLQ-C30. Otolaryngol Head Neck Surg 116(6 Pt 1): 666-673

39. Linden W, Vodermaier A, Mackenzie R, Greig D (2012) Anxiety and depression after cancer diagnosis: Prevalence rates by cancer type, gender, and age. J Affect Disord 141(2-3):343-351

40. Dunn KM, Jordan K, Croft PR (2003) Does questionnaire structure influence response in postal surveys? J Clin Epidemiol 56(1):10-16 